\title{
Numerical Study of Elastic Wave Propagation Characteristics in Cracked Rock
}

\author{
Kaifeng Han, Ruiqi Cao \\ College of Optoelectronic Science and Engineering, National University of Defense Technology, Changsha, \\ Hunan 410073, China \\ Email: hankaifeng@nudt.edu.cn
}

Received January 2014

\begin{abstract}
Numerical methods can provide extremely powerful tools for analysis and design of engineering systems with complex factors that are not possible or very difficult with the use of the conventional methods. In this paper, we use the 2-D boundary element method (BEM) program to model elastic wave excited by a point explosive source propagating in cracked rocks. As an example, we consider the typical crack distributions in rocks, both models for the real crack structure are also talked about. The elastic wave propagating in rocks with aligned cracks and parallel fractures is assumed. Effects of different crack parameters, such as crack scale length and crack density are analyzed. Numerical results show that the BEM is a powerful interpretive tool for understanding the complicated wave propagation and interaction in cracked solids.
\end{abstract}

\section{Keywords}

Numerical Study, Elastic Wave, Propagation Characteristics, Cracked Rock, Boundary Element Method

\section{Introduction}

In recent years, the problem of scattering of elastic waves by cracks or inclusions has gained importance especially in seismology and geophysics. There have been many theories in the literatures that attempt to predict effective properties of elastic wave-fields [1] [2], but few are valid when the size of cracks is large compared with a wavelength.

Various numerical methods have been employed to study elastic wave scattering problems, including Maslov theory [3], the finite difference method [4], the finite element method [5], the Born approximation [6], the complex screen approach [7], Kirchhoff-Helmholtz integration [8], pseudo-spectrum method [9] and the boundary element method [10]. Among these methods, the boundary element method (BEM) is based on a boundary integral equations form required only a mesh of the boundary in a given domain, which means the problem size has been reduced by one dimension and only data in the boundary are calculated. This method has been successfully applied to solve various types of elastodynamic problems in engineering and seismology.

In this paper, a two-dimensional BEM based on workstation or personal computer is developed to modeling the elastic wave propagation in anisotropic and/or heterogeneous medium. The program could be adapted easily 
to three-dimensional case but will require a long computer time and large storage memory. We first compute synthetic seismograms for the case without any cracks to check the accuracy of the program. Our results for synthetic seismograms are achieved in time-domain by means of the inverse Fourier transform of the solution at many frequencies.

\section{Boundary Element Method}

We consider a planar crack $D$ with boundary $R$ in a 2-D unbounded domain subjected to an incident wave $u^{0}$ and assume that the elastic source is located somewhere in the medium outside the crack as Figure 1. The complete displacement wave field $u$ is then written as the superposition of the incident wave-field $u^{0}$ and the scattered wave-field $u^{s}$ :

$$
\boldsymbol{u}=\boldsymbol{u}^{s}+\boldsymbol{u}^{0}
$$

Using displacement Green's function, the scattered wave-field is represented as:

$$
u_{i}^{s}(x)=\int_{L} \Phi_{j}\left(x^{\prime}\right) G_{i j}\left(x, x^{\prime}\right) d L^{\prime}, \quad i, j=1,2,3
$$

where $\boldsymbol{u}_{i}^{\boldsymbol{s}}(\boldsymbol{x})$ is the $i$-th displacement component of scattered waves at any point $\boldsymbol{x}$; and $\boldsymbol{\Phi}_{j}\left(\boldsymbol{x}^{\prime}\right)$ is the strength of the source located at any point $x^{\prime}$ on the boundary $R$ of the crack with outwards normal $\boldsymbol{n}$. $G_{i j}\left(x, x^{\prime}\right)$ is the displacement Green's tensor.

Similarly, using the traction Green's function, the traction representation can be expressed as:

$$
t_{i}(x)= \pm \frac{1}{2} \delta_{i j} \Phi_{j}(x)+\int_{L} \Phi_{j}\left(x^{\prime}\right) F_{i j}\left(x, x^{\prime}\right) d L^{\prime}
$$

where the "+" and "-" signs correspond to approach the boundary of the crack from the interior and exterior respectively. $F_{i j}\left(x, x^{\prime}\right)$ is the traction Green's tensor.

\section{Numerical Examples and Discussions}

The inclusions studied here are circular and elliptical cracks, distributed in full spaces. We modeled 2-D elastic waves excited by a point explosive source $x_{i s}(i=1,3)$ that centers at $(30 \mathrm{~m}, 160 \mathrm{~m}) .65$ receivers are in aligned in the $x_{3}$-direction at $x_{1}=120 \mathrm{~m}$, starting at $x_{3}=320 \mathrm{~m}$ and with an increment of $\Delta x_{3}=-5 \mathrm{~m}$. The source time functions of displacement field are introduced as:

$$
\left\{\begin{array}{l}
u_{1}\left(x_{1}, x_{3}, t\right)=g_{1}\left(x_{1}, x_{3}\right) f(t) \\
u_{2}\left(x_{1}, x_{3}, t\right)=0 \\
u_{3}\left(x_{1}, x_{3}, t\right)=g_{3}\left(x_{1}, x_{3}\right) f(t)
\end{array}\right.
$$

where

$$
\begin{gathered}
g_{i}\left(x_{1}, x_{3}\right)=\left(x_{i}-x_{i s}\right) \exp \left\{-a\left[\left(x_{1}-x_{1 s}\right)^{2}+\left(x_{3}-x_{3 s}\right)^{2}\right]\right\}, \quad i=1,3 \\
f(t)=\left(t-t_{0}\right) \exp \left\{-\lambda\left(t-t_{0}\right)^{2}\right\}
\end{gathered}
$$

Here $a=0.1$ and $\lambda=1500$ are control parameters.

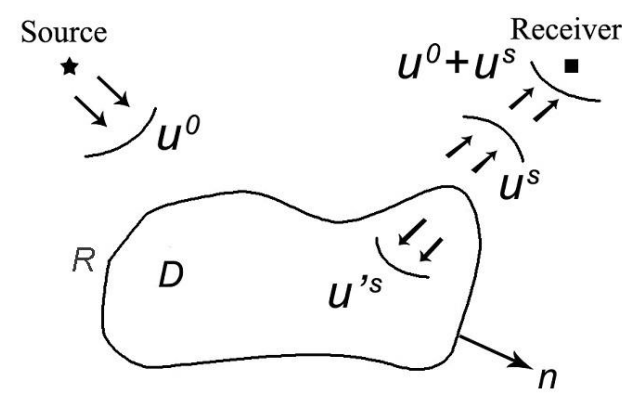

Figure 1. Configuration for the BEM. 
Figure 2 shows the wave propagation in the elastic solid without cracks, which means the inclusion material has the same properties as the surrounding medium, so only the incident wave is produced and the wave speed is independent of the direction. Excellent agreement was achieved between our results and the theoretical results.

The synthetic seismograms of the scattering due to a circular crack in a full-space are shown in Figure 3. The center of the crack is located at $(90 \mathrm{~m}, 160 \mathrm{~m})$, the crack has a radius of $2.5 \mathrm{~m}$ and its boundary is discretized into 30 elements. The crack surrounding solid has $\lambda_{1}=25.168 \mathrm{Gpa}, \mu_{1}=12.584 \mathrm{Gpa}$, and density $\rho_{1}=2600 \mathrm{~kg} / \mathrm{m}^{3}$, and the elastic inclusion has $\lambda_{2}=6.417 \mathrm{Gpa}, \mu_{2}=5.175 \mathrm{Gpa}$, and density $\rho_{2}=2300 \mathrm{~kg} / \mathrm{m}^{3}$. Upon hitting the boundary of the crack, the incident wave is scattered, as is shown in Figure 3. We can find that the scattering waves reaches the receivers with a time delay proportional to their creeping path along the boundary of the crack and the path from the boundary to the receivers.

When there are many cracks in the rock, the problem is too difficult to deal with deterministically but it will be simply treated by statistical methods. The model geometry used to generate the synthetic seismograms is shown in Figure 4. The source, receivers and fracture are situated in an elastic $(\alpha=5800 \mathrm{~m} / \mathrm{s}, \beta=3340 \mathrm{~m} / \mathrm{s}$, $\rho=2600 \mathrm{~kg} / \mathrm{m}^{3}$ ) full space. Two groups of receivers are located at $x_{1}=0$, and $x_{3}=0$ with $50 \mathrm{~m}$ spacing interval respectively.

In this model, crack is placed on a uniform rock matrix. As shown in Figure 4, cracks are placed isolated at pre-assumed location. Two different shapes of cracks are considered here. First, we placed the cracks at only one isolated grid point (Figure 4(a)). This intends to model the case where there is no directional effect. Then, we placed the cracks using 3 grid points (all along the $x_{1}$ direction as shown in Figure 4(b)) as a group. This way of arrangement makes the medium has an anisotropic property. We investigated the response of the medium to two sources with different peak frequencies. For source 1, the peak frequency is $1 \mathrm{~Hz}$. In this case, the wavelength is much longer than the crack size. For source 2, the peak frequency is $50 \mathrm{~Hz}$; the corresponding wavelength is about the same of the crack size. For the reason of easy comparison, the crack density is fixed at 0.12 in all case.

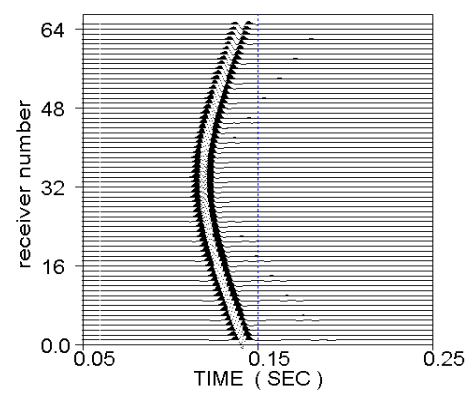

(a)

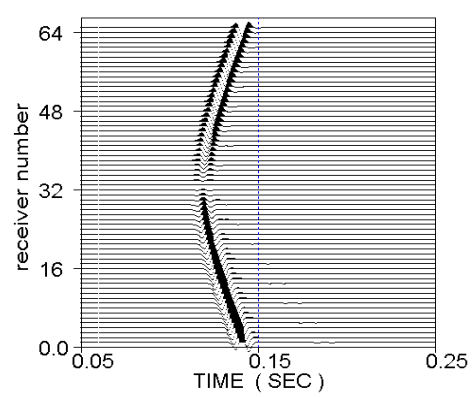

(b)

Figure 2. Elastic waves excited by a point explosive source propagating in the medium without any crack. (a) the horizontal displacement component $\left(u_{1}\right)$; (b) the vertical displacement component $\left(u_{3}\right)$.

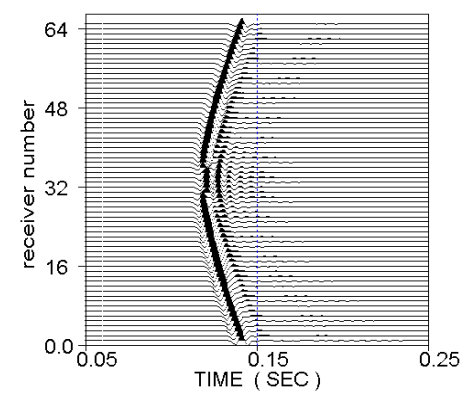

(a)

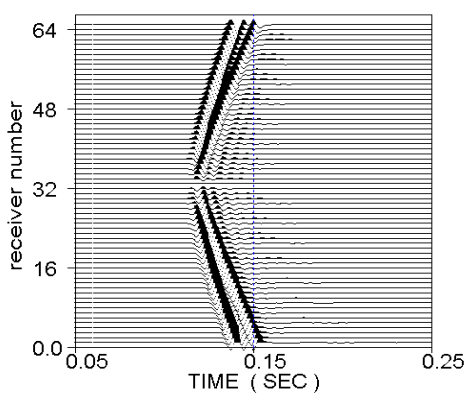

(b)

Figure 3. Elastic waves excited by a point explosive source propagate in the medium containing one circular crack. (a) the horizontal displacement component $\left(u_{1}\right)$; (b) the vertical displacement component $\left(u_{3}\right)$. 
Figure 5 and Figure 6 give the results for source 1 and 2 for the model 4a. As expected, the results are isotropic. In the low frequency case, several wave propagating events could be identified, although scattering is observed. On the other side, for the higher frequency case, as the wavelength is in the same order with the crack size, scattering of elastic wave dominates the propagation process. It is hard to identify any event in the wave field except the beginning of the wave propagating.

Figure 7 and Figure 8 give the results for source 1 and 2 for the model 4b. Comparing Figure 7 with Figure 5, the P-wave velocity has shown the character of anisotropic medium, which agrees well with the theoretical result. Figure 8 is again dominated by the scattering. The P-wave velocities are different along the $x_{1}$ and along the $x_{3}$ direction.

\section{Conclusions}

In this paper, we modeled 2-D elastic waves excited by a point explosive source propagating in the media containing some inclusions by 2-D BEM code. The inclusions studied here are circular and elliptical cracks, distributed in full spaces. For the convenience, we assume that all circular cracks are identical. We have compared our results for the wave propagating in the elastic solid without cracks with the exact analytic solutions to test the accuracy of our program, and then obtained satisfactory solutions.

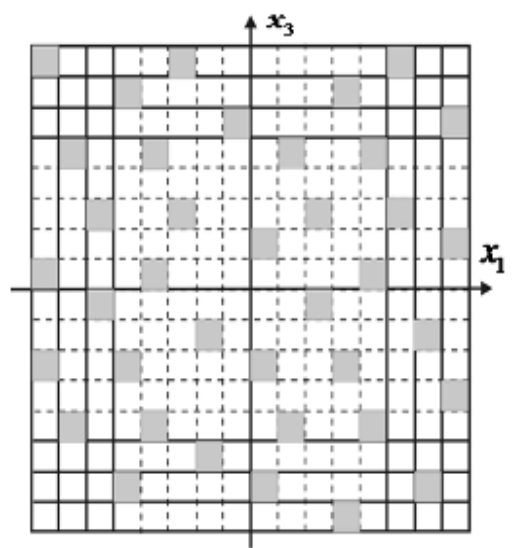

(a)

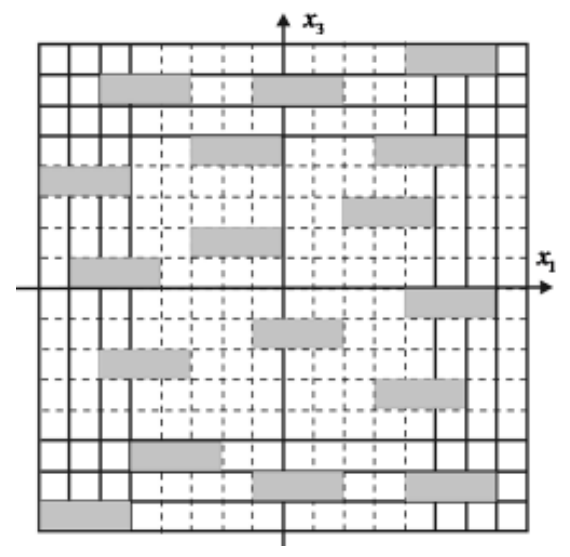

(b)

Figure 4. Illustration for the model. (a) the case when a crack occupied only one single isolated grid; (b) the case when a crack occupied three connected grids in the $x_{1}$ direction.
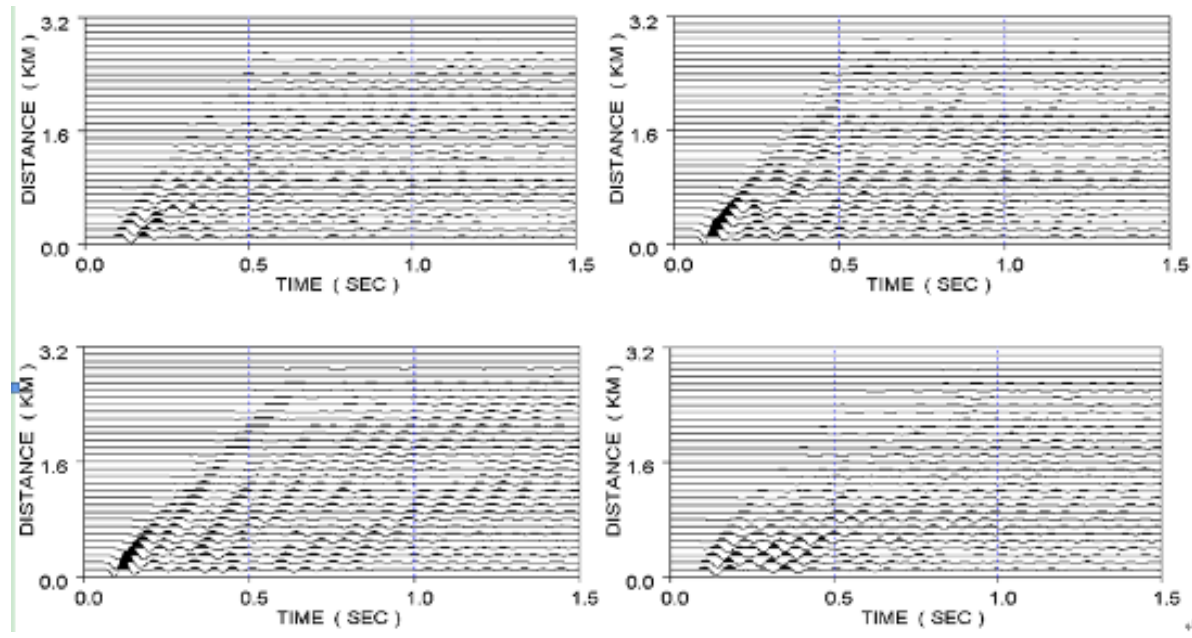

Figure 5. Seismograms for the dry cracks calculated using model $4 \mathrm{a}$. The peak frequency for the source is $1 \mathrm{~Hz}$. 

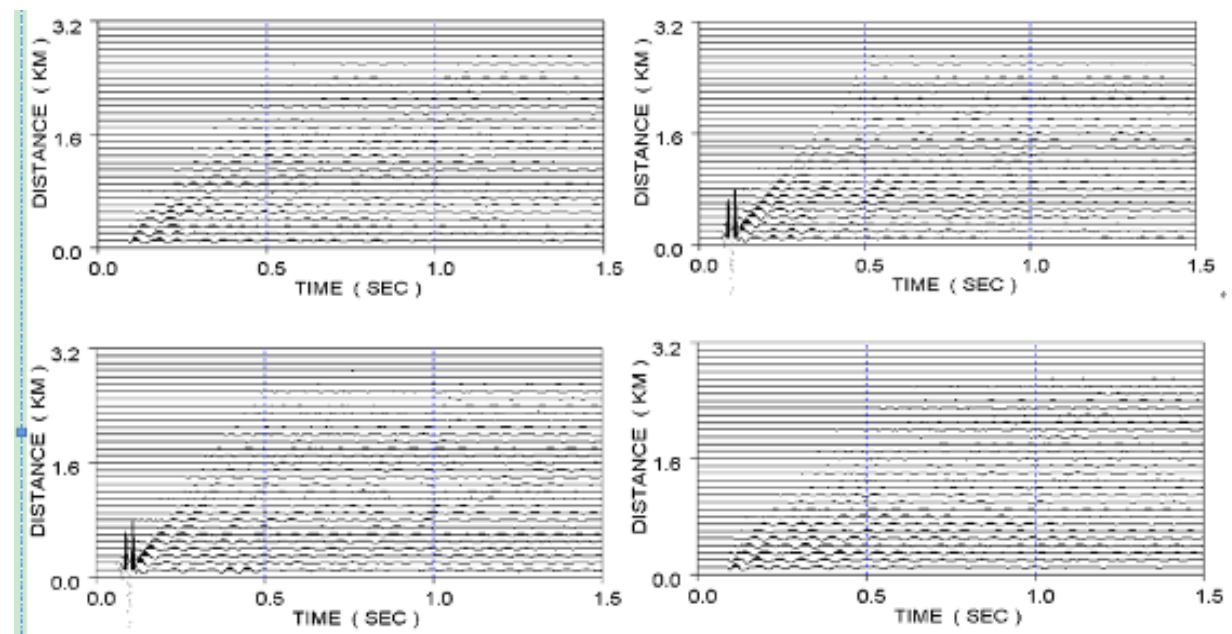

Figure 6. Seismograms for the dry cracks calculated using model 4a. The peak frequency for the source is $50 \mathrm{~Hz}$.
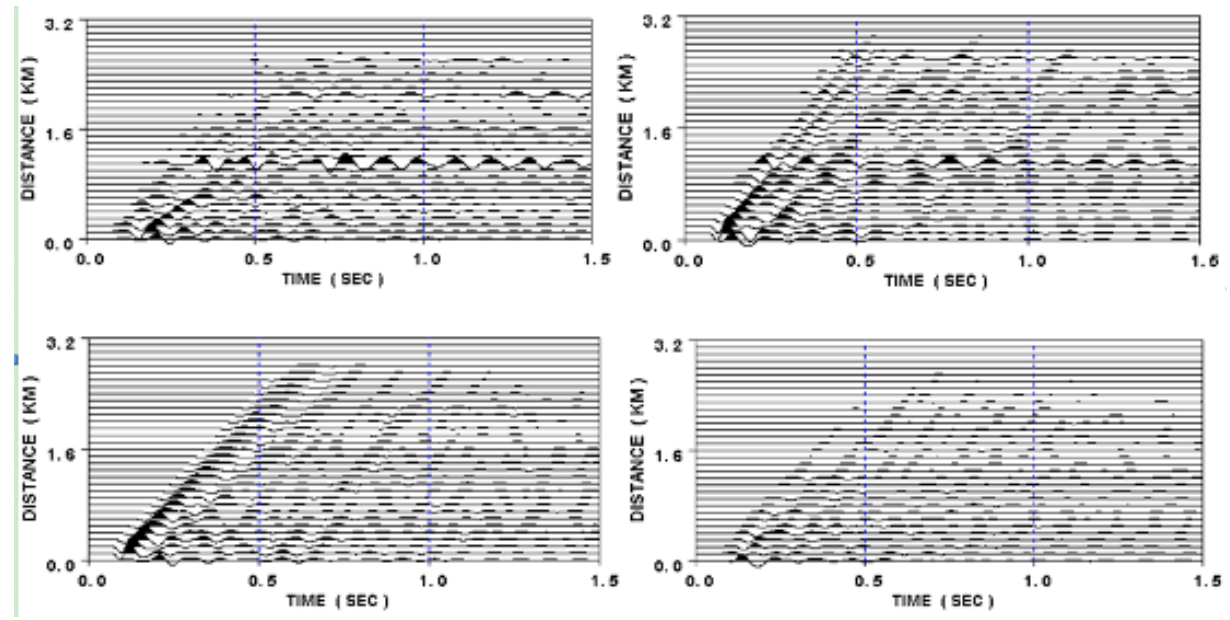

Figure 7. Seismograms for the dry cracks calculated using model $4 \mathrm{~b}$. The peak frequency for the source is $1 \mathrm{~Hz}$.
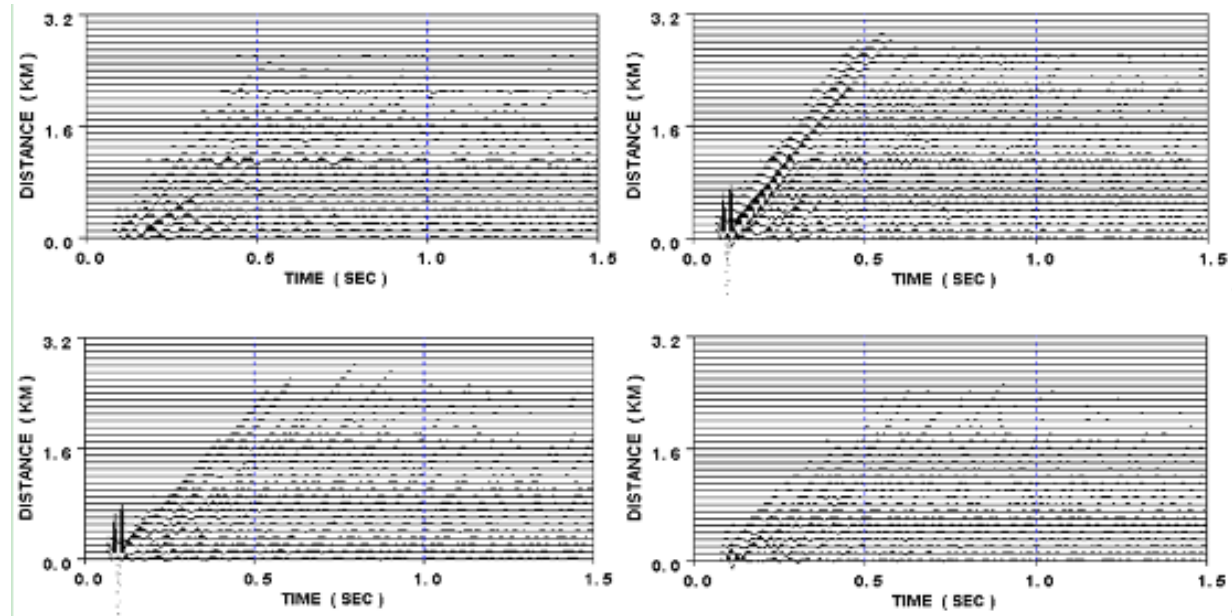

Figure 8. Seismograms for the dry cracks calculated using model $4 \mathrm{~b}$. The peak frequency for the source is $50 \mathrm{~Hz}$. 
In order to study the effects of relative relation for the interface of the cracks, we started with one circular crack; we have found that there are diffracted waves, including reflected, transmitted, converted waves. In the case of two cracks, there exists not only the arrivals due to two cracks respectively but also the arrivals correspond to the waves reflected between the boundaries of the two cracks. In the study of more cracks, we will be interested in the statistical characteristics of the scattered waves instead.

Numerical test shows that the BEM is applicable. We used different models to calculate the seismograms for elastic wave propagating in cracked rocks. The results show that scattering of elastic waves in fractured materials could be modeled by placing the crack in a uniformed rock matrix using the BEM. This primary result only shows the applicable of the method and the result is basically agreed with the condition of long wave approximation. Further researches required to get the quantitative results due to the different crack distributions.

\section{Acknowledgements}

This research is supported by the National Natural Science Foundation of China (No. 41004055 and No. 41374005). This paper is published with the approval of the National University of Defense Technology of China.

\section{References}

[1] Maultzsch, S., Chapman, M., Liu, E. and Li, X.Y. (2003) Modeling Frequency-Dependent Seismic Anisotropy in Fluid-Saturated Rock with Aligned Fractures: Implication of Fracture Size Estimation from Anisotropic Measurements. Geophysical Prospecting, 51, 381-392. http://dx.doi.org/10.1046/j.1365-2478.2003.00386.x

[2] Hudson, J.A. (1986) A Higher Order Approximation to the Wave Propagation Constants for Cracked Solid. Geophysical Journal of the Royal Astronomical Society, 87, 265-274. http://dx.doi.org/10.1111/j.1365-246X.1986.tb04556.x

[3] Chapman, C.H. and Drummond, R. (1982) Body-Wave Seismograms in Inhomogeneous Media Using Maslov Asymptotic Theory. Bulletin of the Seismological Society of America, 72, 277-317.

[4] Fehler, M. and Aki, K. (1978) Numerical Study of Diffraction of Plane Elastic Waves by a Finite Crack with Application to Location of a Magma Lens. Bulletin of the Seismological Society of America, 68, 573-598.

[5] Lysmer, J. and Drake, L.A. (1972) A Finite Element Method for Seismology. Methods of Computational Physics, Academic Press, New York, 11.

[6] Wu, R.S. and Aki, K. (1985) Scattering Characteristics of Elastic Wave by an Elastic Heterogeneity. Geophysics, 50, 582-589. http://dx.doi.org/10.1190/1.1441934

[7] Wu, R.S. (1994) Wide-Angle Elastic Wave One-Way Propagation in Heterogeneous Media and an Elastic Wave Complex-Screen Method. Journal of Geophysical Research, 9, 751-766. http://dx.doi.org/10.1190/1.1441934

[8] Neuberg, J. and Pointer, T. (1995) Modelling Seismic Reflections from D" Using the Kirchohoff Method. Physics of the Earth and Planetary Interiors, 90, 273-281. http://dx.doi.org/10.1016/0031-9201(95)05089-T

[9] Zhang, G.Y. and Zeng, X.W. (2002) Numerical Modeling of Elastic Waves Propagating in the Anisotropic/Heterogeneous Media by the Pseudo-Spectral Method. Journal of National University of Defense Technology, 24, 18-22.

[10] Han, K.F. and Zeng, X.W. (2006) Using Boundary-Element Numeric Simulation to Prove Hudson Fractural Medium Theory. Oil Geophysical Prospecting, 41, 534-540. 